\title{
The Finite Number of Interior Component Shapes of the Levy Dragon
}

\author{
Eytan Alster
}

Received: 11 November 2008 / Revised: 22 June 2009 / Accepted: 25 June 2009 /

Published online: 11 July 2009

(C) Springer Science+Business Media, LLC 2009

\begin{abstract}
The Levy dragon is a connected self-similar tile with disconnected interior. It was previously known that there are at least 16 different shapes of its interior components. Using simple properties of an infinite sequence of curves which converge into the Levy dragon, it is proved that the number of different shapes of the interior components is finite. A detailed description of the buildup of those shapes as unions of various contractions of three convex polygonal shapes is given, and the number of shapes is determined.
\end{abstract}

Keywords Levy dragon · Interior component shapes

\section{Introduction}

The Levy dragon $K$ (Fig. 1) is the attractor of an iterated function system $F=$ $\left\{f_{0}, f_{1}\right\}$ where the two $\mathbb{R}^{2} \rightarrow \mathbb{R}^{2}$ contracting similarity transformations $f_{0}$ and $f_{1}$ include contraction by $1 / \sqrt{2}$ and a rotation by $\pi / 4$ for $f_{0}$ and by $-\pi / 4$ for $f_{1}$. As proved by Huchinson [5], the attractor of such a system is a unique compact set that satisfies

$$
K=f_{0}(K) \cup f_{1}(K)
$$

For any compact set $S$, let $F(S)=f_{0}(S) \cup f_{1}(S)$; then $K=F(K)$, so $K$ is the fixed point of $F$, and

$$
K=\lim _{k \rightarrow \infty} F^{k}(S)
$$

If $S$ is a straight line segment $C_{0}$ whose end points are the fixed points of $f_{0}$ and $f_{1}$, the curves $C_{k}=F^{k}\left(C_{0}\right)$ converge (with respect to the Hausdorff metric) as $k \rightarrow \infty$

E. Alster $(\bowtie)$

Hayarden 45, Ramat Gan 52271, Israel

e-mail: ealster@hotmail.com 
Fig. 1 The Levy dragon
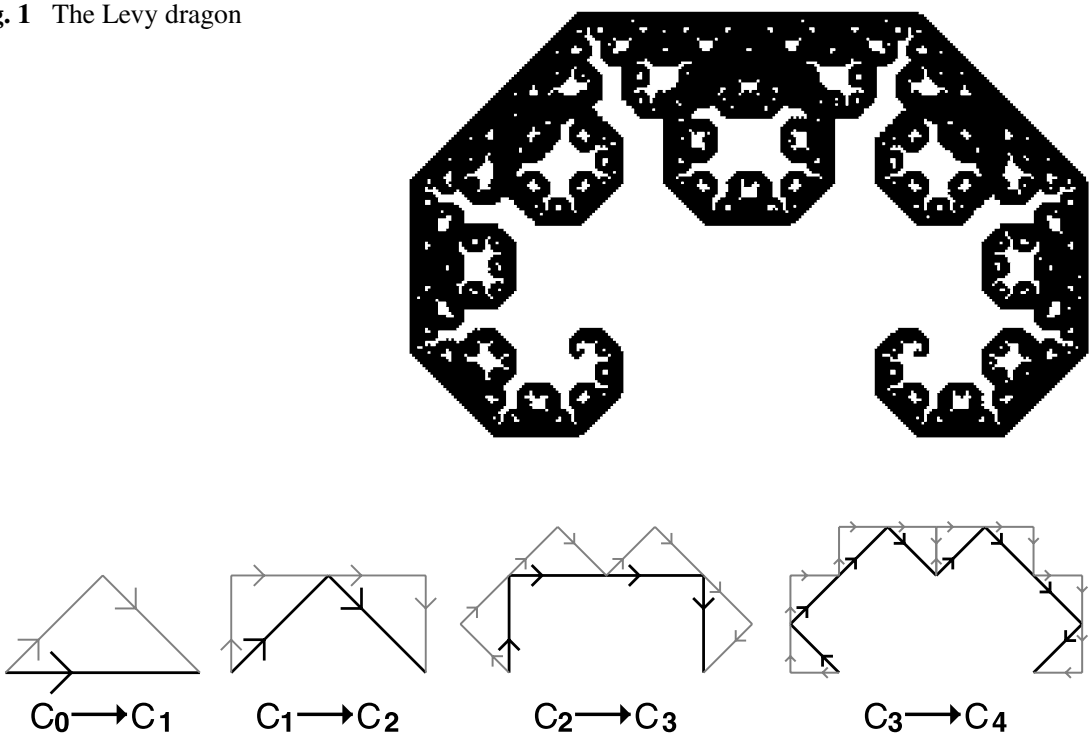

Fig. 2 First $C_{k}$ curves iterations

to $K . C_{k}$ consists of $2^{k}$ segments of length $l_{k}=(1 / \sqrt{2})^{k} l_{0}$, where $l_{0}$ is the distance between the fixed points of $f_{0}$ and $f_{1}$. Figure 2 demonstrates the first iterations in this sequence with a curve in black and its next iteration in gray. Every $C_{k}$ segment has a direction starting with the direction of $C_{0}$ from the fixed point of $f_{0}$ to the fixed point of $f_{1} . C_{k+1}$ is obtained from $C_{k}$ by replacing every segment of $C_{k}$ by two segments that form with it an isosceles right triangle with the $C_{k}$ segment its hypotenuse and the triangle to the left with respect to its direction. The directions of the two $C_{k+1}$ segments replacing a $C_{k}$ segment are such that they form a path from its start to its end. For the rest of this paper, a $C_{k}$ segment means a directed segment as marked by the arrows in Fig. 2. When there are two opposite arrows on the same segment as for one of the segments in $C_{4}$, there are two overlapping segments with opposite directions. Properties of the curves $C_{k}$ are discussed in Sect. 2.

Another choice for $S$ used in many treatments of the Levy dragon is the isosceles right triangle $T$ with end points of the hypotenuse at the fixed points of $f_{0}$ and $f_{1}$ and the third vertex to the left with respect to the direction from the fixed point of $f_{0}$ to the fixed point of $f_{1}$. That third vertex is the only common point of the two contracted triangles in $F(T)$, and their hypotenuses are on two sides of $T$ (Fig. 3(a)). The $2^{k}$ contracted triangles in $F^{k}(T)$ are connected, but their interiors never overlap since, as demonstrated in Fig. 3(b), for any square with up to four $F^{k}(T)$ triangles, the $F^{k+1}(T)$ triangles that replace them are within that square, and their interiors do not overlap.

These two choices for $S$ are thus related: the hypotenuses of the $F^{k}(T)$ triangles are the $C_{k}$ segments, while their other two sides are the $C_{k+1}$ segments. In this paper properties of the curves $C_{k}$ are used through their relation to the $F^{k}(T)$ triangles to determine the shapes of the interior components of the Levy dragon in Sect. 3. 


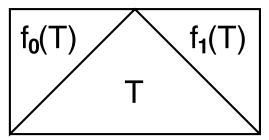

(a)

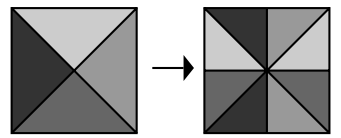

(b)

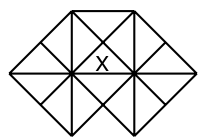

(c)

Fig. 3 (a) Triangle $T$ and its two contractions $f_{0}(T)$ and $f_{1}(T)$. (b) Four triangles in a square and their next iteration. (c) A covered triangle

A similar method was used by Ngai and Nguyen [7] to prove geometric properties of the Heighway dragon using properties of the dragon curves.

P. Levy introduced in 1938 [6] (annotated English translation in [4]) the set now known as the Levy dragon as the limit of the sequence of curves $C_{k}$ within a discussion of the properties of self similar curves like the Koch curve. Many properties of the Levy dragon are proved in [6]. Among them the location and shape of the largest of the interior components that consists of 22 triangles of $F^{16}(T)$ hence covers $22 / 2^{16} \approx 1 / 3000$ of the area of the Levy dragon, which is equal to the area of triangle $T$.

The Hausdorff dimension of the boundary of the Levy dragon was first calculated by Duvall and Keesling [2] to be $D=1.934007183 \ldots$. This dimension was calculated by Strichartz and Wang [9] using a different method.

Deng and Ngai [3] calculated the dimensions of subsets of the boundary of the Levy dragon which are the common intersection of at least a given number of neighbors in the tiling of the plane by copies of the Levy dragon. Ngai and Tang [8] proved that the closure of each interior component of the Levy dragon is a topological disk.

An $F^{k}(T)$ triangle with all the 14 triangles intersecting it also in $F^{k}(T)$, as demonstrated in Fig. 3(c) for triangle $X$ there, is called in [2] covered since it is tiled by two $F^{k+1}(T)$ covered triangles and thus is a subset of $K$.

The Levy dragon is a connected set with disconnected interior. Bailey, Kim, and Strichartz [1] showed that contrary to the dragon boundary being a fractal, each of the infinitely many interior components, which are all too small to be seen in Fig. 1, has a one-dimensional polygonal boundary. By using a computer program that acts as a "microscope" into $F^{k}(T)$ and differentiates between an $F^{k}(T)$ triangle that is covered or not, the buildup of the interior components is observed. Covered triangles appear for the first time in $F^{14}(T)$, and following the growth of connected sets of covered triangles, they observed that distinct sets of covered triangles never merge and that some covered sets of triangles stop growing after a few iterations while other sets never stop growing. They identified 16 shapes of interior components and conjectured that each interior component is similar to one of those shapes.

\section{Properties of the Curves $C_{k}$}

The points where $C_{k}$ segments are joined are called its vertices. By the iteration process every vertex of $C_{k}$ is also a vertex of any $C_{k+m}$ and hence is in $K$. Every vertex has a type which describes the direction change of the segment after it with respect to the segment before it. For two consecutive segments in $C_{k}$, if the directions of the segments before and after the vertex are $\alpha$ and $\beta$, respectively, then the type of 


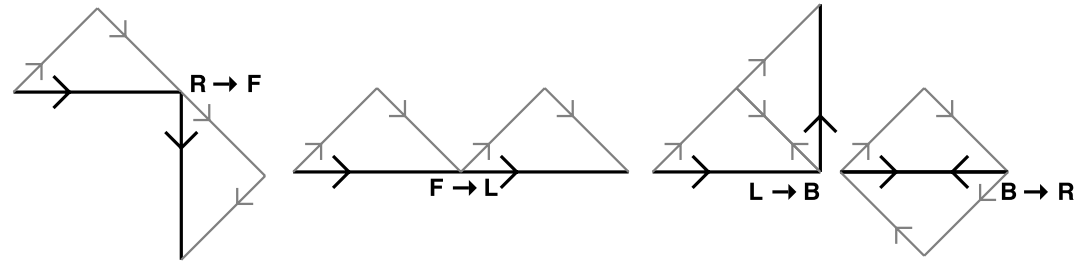

Fig. 4 The change of vertices type after one iteration

the vertex is $\beta-\alpha$. In $C_{k+1}$ the segment before that vertex is the second segment out of the two that replace the $C_{k}$ segment before it, and hence its direction is $\alpha-\pi / 4$, while the segment after that vertex is the first segment out of the two that replace the $C_{k}$ segment after it, and hence its direction is $\beta+\pi / 4$. The type of that $C_{k+1}$ vertex is thus $(\beta+\pi / 4)-(\alpha-\pi / 4)=(\beta-\alpha)+\pi / 2$. The type of a vertex changes by a right angle after every iteration, and after four iterations it returns to the same type.

There are $2^{k-1}$ new vertices in $C_{k}$, one for every segment of $C_{k-1}$. The type of all of those new vertices is $\pi / 2$ to the right since a $C_{k-1}$ segment is replaced by two $C_{k}$ segments to its left. There are thus only four types denoted by $R$ for a turn to the right, $F$ for continuing forward, $L$ for a turn to the left, and $B$ for a turn backward. As demonstrated in Fig. 4, a vertex created with type $R$ in $C_{k}$ has type $F$ in $C_{k+1}$, type $L$ in $C_{k+2}$, type $B$ in $C_{k+3}$, and it returns to type $R$ in $C_{k+4}$. Every vertex thus goes through a cyclic change of type with period 4 along the sequence of iterations. A vertex created in $C_{k}$ is $R$ type in $C_{k+4 m}, F$ type in $C_{k+4 m+1}, L$ type in $C_{k+4 m+2}$, and $B$ type in $C_{k+4 m+3}$ for any nonnegative integer $m$. The type of a vertex with type $X$ that was created in $C_{k-m}$ is denoted by $X^{[k-m]}$.

At one of the ends of any $C_{k}$ segment there is a vertex with type $R^{[k]}$. The vertex at the other end was created by $C_{k-m}$ for some $m>0$. If two $C_{k}$ segments overlap, they must have opposite directions because if two overlapping segments have the same direction, then at the end where the vertices with type $R^{[k]}$ are, there are two other overlapping segments with the same direction. This could happen only if there were two overlapping segments with the same direction in $C_{k-1}$. Continuing backward along the iterations sequence and repeating this argument would lead to the existence of overlapping segments pair with the same direction in any $C_{k-m}$. However, $C_{0}$ (and also $C_{1}, C_{2}$, and $C_{3}$ ) do not have overlapping segments at all, and hence there are no overlapping segments with the same direction in any $C_{k}$. Thus, no more than two segments can overlap because a third would have the same direction as one of them. For the rest of this paper, such an overlapping segments pair is referred to as an OSP. Another corollary of this is that no more than four vertices are located at the same point.

Since all the segments of $C_{k}$ have the same length and all the turns at the vertices are multiples of right angles, all the segments of $C_{k}$ are on the sides of squares of a lattice of squares tiling $\mathbb{R}^{2}$. These squares are referred to as the $C_{k}$ squares. All the new vertices created by the next iteration are at centers of those squares and the $C_{k+1}$ squares are obtained by the diagonals of the $C_{k}$ squares. Hence, all the vertices located at one point were created by the same iteration as $R$ type and thus have the same type after any number of additional iterations. 
If there are vertices on the four corners of a $C_{k}$ square, then the vertices on two opposite corners were created by $C_{k}$ at centers of two adjacent $C_{k-1}$ squares and thus have type $R$. The other two opposite corners are at the two ends of one side of a $C_{k-1}$ square, and if there was a $C_{k-1}$ segment on it, on one of its ends there would be a vertex created as $R$ by $C_{k-1}$ and thus would have type $F$ in $C_{k}$. Hence, the vertices on two opposite corners of a $C_{k}$ square are $R^{[k]}$ and on one of the other corners $F^{[k-1]}$. The vertex at the last corner was created by some iteration up to $C_{k-2}$.

Once the types of the vertices in a neighborhood are known, one can determine which segments are in the same path within that neighborhood. The segments in the same path either all exist or all are absent.

\section{The Shapes of Interior Components}

Let $P$ be the reflection through the line perpendicular to $C_{0}$ at its center. Then $P^{2}$ is the identity, and since the fixed points of $f_{0}$ and $f_{1}$ are at the ends of $C_{0}$ and their rotation angles are opposite, $P f_{0} P=f_{1}$ and $P f_{1} P=f_{0}$, and thus

$$
P(K)=P f_{0}(K) \cup P f_{1}(K)=P f_{0} P^{2}(K) \cup P f_{1} P^{2}(K)=f_{1} P(K) \cup f_{0} P(K)
$$

and since the attractor of the iterated function system $\left\{f_{0}, f_{1}\right\}$ is unique [5], we have $P(K)=K$. Hence, $P$ is a symmetry of the Levy dragon, and thus for every shape of interior component, the reflected shape also is a shape of an interior component. We consider such a pair of reflected shapes as one shape.

To find the shapes of the interior components, we start with the concept of a covered triangle, as demonstrated in Fig. 3(c), and transfer it to the segment of $C_{k}$ so that the hypotenuse of the covered triangle is on it and the triangle is to its left. Thus, the minimal configuration for a covered segment is as in Fig. 5(a) for segment $s$ there. It includes seven OSPs and one single segment, which is the hypotenuse of the triangle that intersects the covered triangle only at its right angle vertex. The opposite segment to a covered segment is almost covered, missing only this last single segment. Since there is an $R$ vertex at one of the ends of this single segment because of the existence of one of the segments in the OSPs perpendicular to the single segment, the segment opposite to it must also exist. Also, because there is an $R$ vertex at the end of one of the two OSPs to the right of segment $s$, there is a segment antiparallel to $s$ there too. Thus, the minimal configuration for a covered segment $s$ is as in Fig. 5(b).

The significance of covered segments for the purpose of finding the shapes of the interior components is that if a $C_{k}$ segment is covered, the two $C_{k+1}$ segments that are opposite to the two segments that replace it are also covered. See Fig. 5(c), where the $C_{k+1}$ segments (in gray) that replace the $C_{k}$ segments (in black) are shown, and segments $r$ and $t$ are covered since $s$ is covered. Thus, the $F^{k}(T)$ triangle to the left of the covered segment is tiled by two covered $F^{k+1}(T)$ triangles. Continuing to the next iterations, for any nonnegative integer $m$, this triangle is tiled by $2^{m}$ triangles of $F^{k+m}(T)$; hence a covered triangle is a subset of $K$, and its interior is a subset of the interior of $K$.

We say that two $F^{k}(T)$ triangles are edge connected when they have a common edge. The interior of the union of a set of edge connected covered triangles is in the 


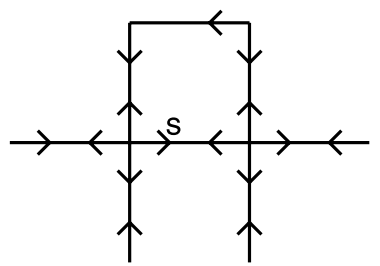

(a)

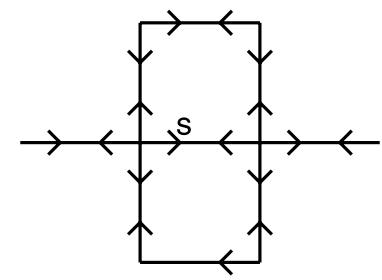

(b)

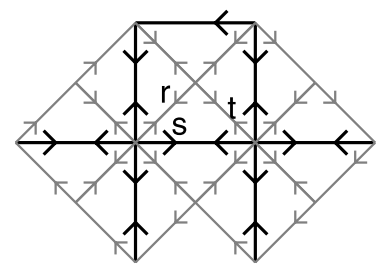

(c)

Fig. 5 (a) The segments configuration of a covered segment $s$. (b) The necessary extra segments. (c) $C_{k+1}$ segments $r$ and $t$ are covered since $C_{k}$ segment $s$ is covered

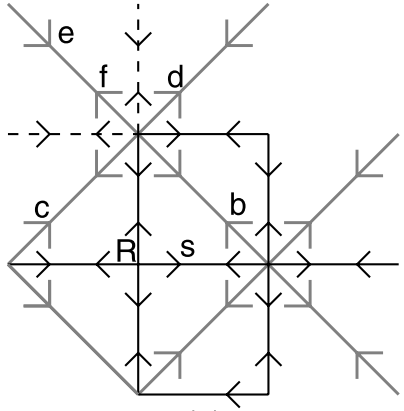

(a)

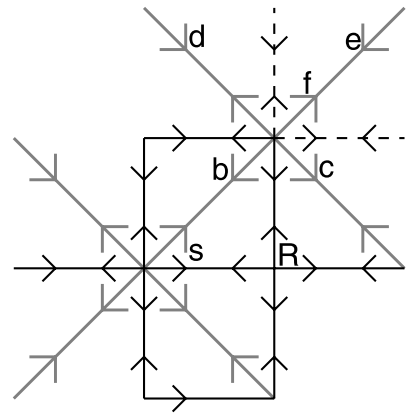

(b)

Fig. 6 The two configurations of a $C_{k}$ segment $s$ originally covered since $C_{k-1}$ segment $b$ is not covered

same interior component of $K$. The buildup of a component of the interior of $K$ during successive iterations of $F^{k}(T)$ must start with the appearance after some iteration of a set of edge connected covered triangles that are not edge connected to any covered triangle generated in previous iterations. Such a seed of an interior component grows into its final shape as in the next iterations there are covered triangles edge connected to it. Finding covered triangles will thus not serve our purpose since they might be tilling a triangle that was already covered in previous iterations. Therefore we define the concept of an originally covered segment for a triangle that is not a subset of a covered triangle.

Definition 3.1 A $C_{k}$ segment is originally covered if it is covered and the opposite segment is one of the two segments that replace a $C_{k-1}$ segment that is not covered.

Figures 6(a) and (b) show the two configurations for a $C_{k}$ covered segment $s$ (in black) with the type $R^{[k]}$ vertex at the beginning and at the end of $s$, four more $C_{k}$ segments in dashed lines and the segments that must be in $C_{k-1}$ for those $C_{k}$ segments to exist (in gray). The four $C_{k-1}$ segments denoted with $c, d, e$, and $f$ must exist if the $C_{k}$ segments in dashed lines exist. Segment $b$ in both cases is the $C_{k-1}$ segment that must be not covered in order that segment $s$ will be originally covered. For $b$ to be not covered, at least one of the four segments $c, d, e$, and $f$ must be absent in $C_{k-1}$, and thus at least one of the four segments with dashed lines is absent in $C_{k}$. 


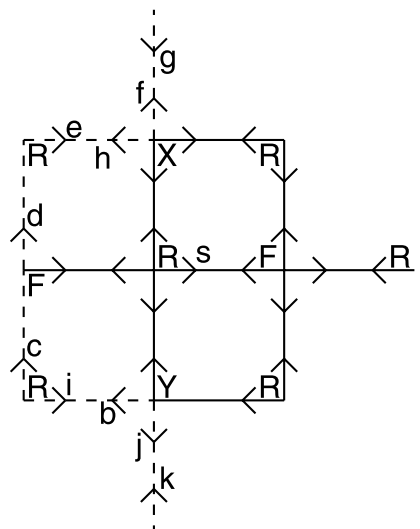

(a)

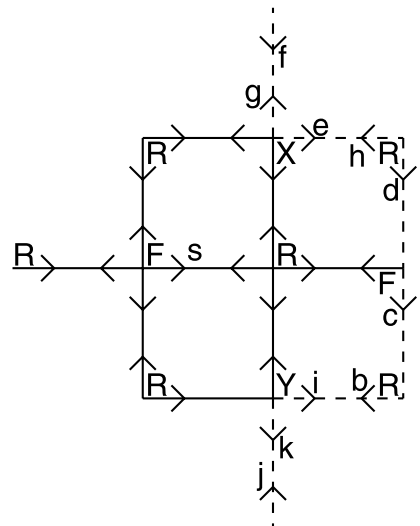

(b)

Fig. 7 The two minimal configurations of an originally covered segment

Lemma 3.2 A $C_{k}$ originally covered segment has a vertex with type $F^{[k-1]}$ at one of its end points.

Proof Let the $C_{k}$ originally covered segment be $s$ in Figs. 6(a) and (b). Consider the $C_{k}$ square to the left of $s$. As any $C_{k}$ square, it has two $R^{[k]}$ vertices on two opposite corners and an $F^{[k-1]}$ vertex on one of the other two corners. In both cases, Figs. 6(a) and (b), the $F$ vertex cannot be at the corner intersected by the four $C_{k}$ segments with dashed lines, since if it does, all those four segments are in paths of the minimal configuration for $s$ to be covered, hence all of them exist, and then $s$ is not originally covered. Thus, the $F^{[k-1]}$ vertex must be on the last corner, which is an end point of segment $s$ in both cases.

By the same argument, also the OSP collinear with $s$ at its $R$ end has an $F^{[k-1]}$ vertex at its other end. The minimal two configurations of an originally covered segment is demonstrated in Figs. 7(a) and (b). The points at the ends of the OSPs perpendicular to $s$ at its $R$ end are denoted $X$ and $Y$ (we use those for the vertex and its type).

Proposition 3.3 The segment opposite to an originally covered segment is covered. If it is originally covered, then there are only three possible configurations:

- $X=B, Y=L$

- $X=L, Y=B$

- $X=L, Y=L$

The segments with end points at $X$ and $Y$ that must be absent are in one path. If it is only covered, there are only two possible configurations:

- $X=B, Y=L$

- $X=R, Y=L$ 
Proof The segment opposite to a covered segment is almost covered, missing only the single segment antiparallel to it on the opposite edge of the square to its left. Consider the originally covered segment $s$ in the two cases in Figs. 7(a) and (b), and let $s^{\prime}$ be the opposite segment. Since $X, Y$, and the two $F^{[k-1]}$ vertices are on the four corners of a $C_{k-1}$ square, either $X$ or $Y$ has type $L^{[k-2]}$.

For both cases, if $Y$ is either $R$ or $F$, then $X=L$, and the segments $h$ and $g$ exist since they are in the paths of segments in the covered configuration, and thus since $s$ is originally covered, both $e$ and $f$ must be absent. But if $Y$ is $R$ or $F$, segment $b$ must exist, and hence the whole path $b, c, d, e$ must exist. Hence, $Y$ is neither $R$ nor $F$. Thus, $Y$ is either $B$ or $L$, and in both cases the one segment needed to make $s^{\prime}$ covered must exist, hence $s^{\prime}$ is covered.

Thus, if $X=L, s^{\prime}$ is originally covered, and either $Y=L$ or $Y=B$. If $X \neq L$, then $Y=L$, and if $s^{\prime}$ is originally covered, then segments $i$ and $j$ exist and segments $k$ and $b$ are absent. Hence, the path that includes segments $b, c, d$, and $e$ is absent. But if $X=R$ or $X=F$, segment $e$ exists, hence $X$ can only be $B$.

The path that includes segments $b, c, d$, and $e$ is absent in both cases, and we refer to it in these cases as the forbidden path.

If segment $s$ is originally covered while $s^{\prime}$ is only covered, then two of the segments $e, f, g$, and $h$ must be absent while all the four segments $i, b, j$, and $k$ must exist. Since segment $b$ exists, all the path $b, c, d, e$ exists. If $X=L$, then $h$ and $g$ exist, and thus $e$ and $f$ must be absent, hence $X \neq L$. Also, $X \neq F$ since then all four segments exist, hence $X$ is either $B$ or $R$. Since $X \neq L$, we have $Y=L$.

We name the three possible configurations of an OSP with the two segments originally covered by the types appearing in clockwise order around the covered area; hence we refer to the three possibilities above as the $L B, B L$, and $L L$ configurations, respectively.

By Proposition 3.3 an originally covered segment can never be part of the boundary of an interior component. The hypotenuse of an originally covered $F^{k}(T)$ triangle is thus never on the boundary of an interior component, and only the two short edges of such a triangle can be part of the boundary. The following proposition characterizes the segments that form the boundary of covered sets.

Proposition 3.4 If a segment is covered and the opposite segment is not covered, then those segments are on the boundary of the covered set which includes the covered triangle to the left of the covered segment.

Proof The configuration for such an OSP in $C_{k}$ with one segment covered and the other not covered is demonstrated in Fig. 5(a), where segment $s$ is the covered segment, and the opposite segment is not covered only because one segment is absent. In $C_{k+2}$ every $C_{k}$ segment is replaced by a $C_{2}$ to its left. On every half of segment $s$ and its opposite, there is a $C_{k+2}$ OSP where the segment with the same direction as $s$ is covered while the opposite segment is not covered. Thus, for all $p>0$, in $C_{k+2 p}$ on segment $s$ and its opposite there are $2^{p}$ OSPs with one segment covered and one not covered, so all the $F^{k+2 p}(T)$ triangles with hypotenuse on $s$ are not covered; hence $s$ is on the boundary of the covered area. 
Fig. 8 (a) The state of segments $s_{R}$ and $s_{F}$ replacing an originally covered segment $s$. (b) The three stages of development of covered area starting with an originally covered OSP

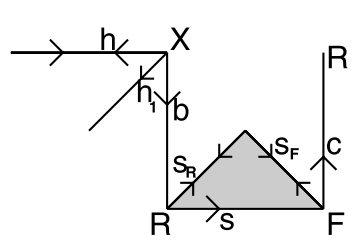

(a)

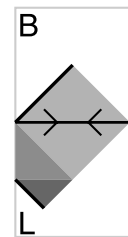

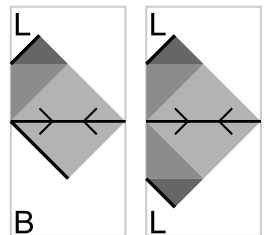

(b)

Lemma 3.5 From the two $C_{k+1}$ segments that replace an originally covered $C_{k}$ segment $s$, one is either originally covered or not covered, while the other is either covered or not covered depending on whether the segment opposite to $s$ is also originally covered or only covered and on the type $X$ at the end of the OSP perpendicular to $S$ at its $R$ end.

Proof Consider Fig. 8(a). Segment $s$ is a $C_{k}$ originally covered segment that starts at the $R$ vertex and ends at the $F$ vertex. The gray triangle is the $F^{k}(T)$ triangle thus covered. Segments $s_{R}$ and $s_{F}$ are the two $C_{k+1}$ segments that replace $s$. By Lemma 3.2, since $s_{R}$ starts at an $F^{[k]}$ vertex and ends at an $R^{[k+1]}$ vertex, it can be originally covered in $C_{k+1}$, while $s_{F}$ ends at an $L$ vertex, and hence it cannot be originally covered.

The segment opposite to $s_{R}$ is covered because $s$ is covered, and therefore $s_{R}$ is almost covered, and it is covered if the $C_{k+1}$ segment $h_{1}$ exists and not covered if $h_{1}$ is absent. For $s_{R}$ to be originally covered, the $C_{k}$ segment $b$ must be not covered. Since $s$ is originally covered, two segments at the $X$ end of $b$ are absent, and thus segment $b$ is not covered; so if $s_{R}$ is covered, it is originally covered. For segment $h_{1}$ to exist in $C_{k+1}$, segment $h$ must exist in $C_{k}$. By Proposition 3.3, since $s$ is originally covered, the opposite segment is covered, and if it is originally covered, then either $X=L, h$ exists, and $s_{R}$ is originally covered or $X=B, h$ is absent, and $s_{R}$ is not covered, hence by Proposition 3.4 it is a boundary segment. If the segment opposite to $s$ is only covered, then by Proposition 3.3 either $X=B, h$ exists, and $s_{R}$ is originally covered or $X=R, h$ is absent, and $s_{r}$ is a boundary segment.

Since $s_{F}$ cannot be originally covered, either it is not covered and part of the boundary of the interior component or it is covered. It can only be covered if the $C_{k}$ segment $c$ is covered. If $c$ is covered, it is originally covered because of the two segments that are absent at $X$ since $s$ is originally covered.

In case $s$ starts at the $F$ vertex and ends at the $R$ vertex, the same arguments lead to the same results, only $s_{R}$ is the second of the two segments replacing $s$, while $s_{F}$ is the first.

Theorem 3.6 $A C_{k}$ OSP with the two segments originally covered develops up to $C_{k+2}$ into one out of three covered shapes in $F^{k+2}(T)$ depending on whether it is an $L B, B L$, or $L L$.

Proof The covered shape for the OSP of two $C_{k}$ originally covered segments is a square tiled by two $F^{k}(T)$ triangles (lighter gray areas in Fig. 8(b)). By Lemma 3.5, 
if $X=L$, then on an edge of that square there is a $C_{k+1}$ OSP with one originally covered segment and one covered segment, so the covered shape grows by an $F^{k+1}(T)$ triangle (darker gray areas in Fig. 8(b)). This happens once for an $L B$ or $B L$ and twice for $L L$. If $X=B$, that $C_{k+1}$ segment is a boundary segment of the covered shape, so for an $L B$ or $B L$, one edge of the square is part of the boundary (solid black lines in Fig. 8(b)). On the other two edges of the covered square, either the OSP has one covered segment and one not covered, so it is a boundary segment, or both are covered when there is another $C_{k}$ originally covered segment perpendicular to the originally covered OSP at its $F$ end.

For the $C_{k+1}$ OSP with one covered segment and one originally covered, when $X=L$, the $R$ vertices are at the beginning of the originally covered one, hence its $X$ point is at the same point as for the $C_{k}$ originally covered OSP, only in $C_{k+1}$ the type turns to $X=B$. By Lemma 3.5 again on one edge of the $F^{k+1}(T)$ triangle that was added, there is a $C_{k+2}$ OSP with one segment covered and the second originally covered, hence the covered shape grows by an $F^{k+2}(T)$ triangle (the darkest gray areas in Fig. 8(b)). The $C_{k+2}$ OSP on the other edge either has one covered segment and one not covered, so it is a boundary segment, or both are covered when there is another $C_{k+1}$ originally covered segment perpendicular to the $C_{k+1}$ originally covered segment at its $F$ end.

Finally for the $C_{k+2}$ OSP with one covered segment and one originally covered, its $X$ point is again at the same point, only in $C_{k+2}$ the type turns to $X=R$. By Lemma 3.5, on one edge of the $F^{k+2}(T)$ triangle that was added, there is a $C_{k+3}$ OSP with one segment covered and the second not covered, hence it is a boundary segment. The $C_{k+3}$ OSP on the other edge either has one covered segment and one not covered, so it is a boundary segment, or both are covered when there is another $C_{k+2}$ originally covered segment perpendicular to the $C_{k+2}$ one at its $F$ end. This originally covered segment exists if there is another $C_{k}$ originally covered segment perpendicular to the originally covered OSP at its $F$ end. Thus, the collinear $C_{k+1}$ and $C_{k+3}$ segments are either both boundary segments or both covered.

To determine the possible interior components shapes, we now first explore the possible seeds of covered areas and then their possible growth patterns.

Theorem 3.7 The possible $C_{k}$ configurations for the initial edge connected set of covered $F^{k}(T)$ triangles of an interior component include only two configurations with one originally covered OSP, four configurations with two originally covered OSPs, and two configurations with four originally covered OSPS.

Proof The first covered triangles of an interior component that appear in $C_{k}$ are not edge connected to triangles that were covered before; hence by Proposition 3.3 they must appear only in OSPs with both segments originally covered.

Consider Fig. 9. It shows the $C_{k}$ segments configuration for an $L B$ originally covered OSP with segments denoted by $s$ and $b$. The square that has the $B$ and $L$ vertices on two adjacent corners and the $F$ end of the originally covered OSP at its center is a $C_{k-2}$ square; hence the type at the corner opposite the $L$ is another $L$. The type at the last corner is denoted by $Z$. The path of forbidden segments is marked 
Fig. 9 The segments configuration of an $L B$ originally covered OSP and the three possible OSPs with edge connected covered squares

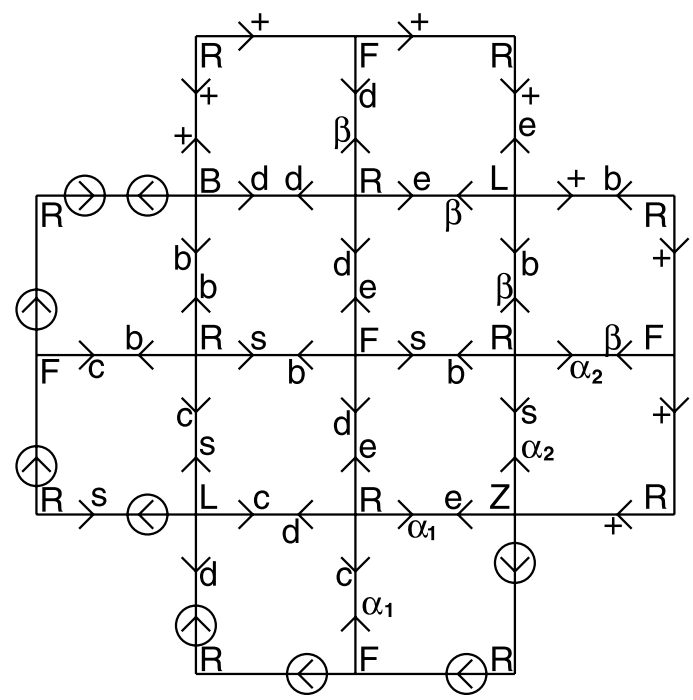

with circled arrows, and it extends up to the point $Z$. There is also a path of segments denoted with a + sign which extends up to $Z$. All the segments in the minimal $L B$ configuration are in five paths, and all the segments in one path are denoted by the same labels $s, b, c, d$, and $e$. Also segments in three additional paths are denoted by $\alpha_{1}, \alpha_{2}$, and $\beta$. The segments in the + path and the $\alpha_{1}, \alpha_{2}$, and $\beta$ paths are not in the minimal configuration of the $L B$ and hence may exist or be absent.

The covered area for an originally covered OSP is a square. For another originally covered OSP to be with a covered square edge connected to this square, it must be an OSP perpendicular to the first with a common end. By Lemma 3.2 such an originally covered OSP must have an $F^{[k-1]}$ at one of its ends. Hence, the only candidates are the two OSPs at the $F$ end with segments $d$ and $e$. For one of them to be originally covered, the $\beta$ path must exist, while the + path must be absent since it is its forbidden path. For the other to be originally covered, the $\alpha_{1}$ and $\alpha_{2}$ paths must exist and either $Z=B$ or $Z=L$ (its forbidden path is part of the forbidden path of the $L B$ ). The only additional possible originally covered OSP with covered square edge connected to the covered squares of those two is the OSP with segments $s$ and $b$ collinear with the first one with common end at its $F$ end. For this OSP to be originally covered, the $\alpha_{1}, \alpha_{2}$, and $\beta$ paths must exist, the + path must be absent, and $Z$ must be either $L$ or $B$. But these are the conditions for the other two OSPs to be originally covered, hence only 1,2 , or 4 originally covered OSPs with edge connected squares can appear together.

Figure 10 demonstrates all these possibilities relative to the $C_{k}$ originally covered OSPs. The gray shapes are obtained by applying Theorem 3.6 in each case, and there are solid black lines on the segments already known by Theorem 3.6 to be boundary segments. Whenever the + path is optional, there is a + near the $B$.

If the $\beta$ path exists, the + path must be absent, because otherwise there is a covered OSP which is not originally covered with its covered square edge connected to the 


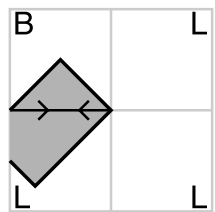

(a)

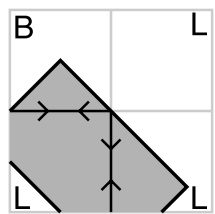

(e)

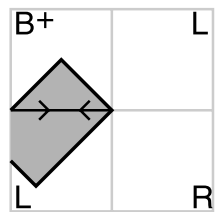

(b)

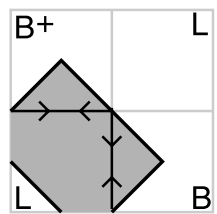

(f)

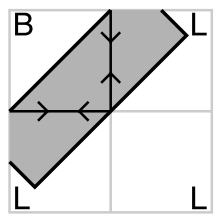

(c)

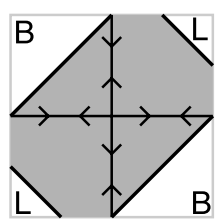

(g)

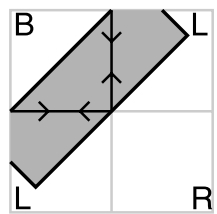

(d)

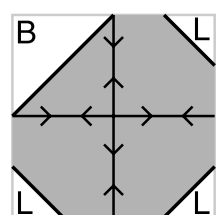

(h)

Fig. 10 The eight possible seeds of interior components

covered square of the first LB, and this is impossible for the initial appearance of covered triangles for an interior component.

$Z \neq F$ because if $Z=F$, the $s$ path is in the forbidden path.

If $Z=R$, then $\alpha_{1}$ is in the forbidden path, and it is absent. Then either the $\beta$ path is absent, it is an LB as in Fig. 10(b), and the + path is optional, or the $\beta$ path exists, the + path is absent, and it is an LBL as in Fig. 10(d).

If $Z=B$, then $\alpha_{1}$ is in the $e$ path and $\alpha_{2}$ is in the $s$ path, so both of them exist. If the $\beta$ path is absent, then it is a BLB as in Fig. 10(f) with the + path optional. If the $\beta$ path exists and the + path is absent, then it is an LBLBL as in Fig. 10(g). (The first $L$ is repeated at the end for a reason that will be clear later.)

If $Z=L$, then the + path is in the forbidden path, and it is absent. $\alpha_{1}$ and $\alpha_{2}$ are in the same path $\alpha$. If both $\beta$ and $\alpha$ are absent, it is an LB as in Fig. 10(a). If $\beta$ exists and $\alpha$ is absent, it is an LBL as in Fig. 10(c). If $\beta$ is absent and $\alpha$ exists, it is an LLB as in Fig. 10(e). If both $\beta$ and $\alpha$ exist, it is an LBLLL as in Fig. 10(h).

There is no need to repeat these arguments for the configurations that start with a BL or LL since these are included within those possibilities rotated or reflected.

The only manner in which these core shapes that are initiated in $C_{k}$ and reach their final shape in $C_{k+2}$ can grow is if in $C_{k+1}$ there is an originally covered OSPs such that the covered squares they generate have an edge on the $C_{k+2}$ segments on the edge of the shape which might not be a boundary segments. In Fig. 11 these OSPs for an $L B, B L$, and $L L$ are denoted with $g$. If the $g$ segments are covered, they are originally covered because the forbidden path prevents the relevant $C_{k}$ segments from being covered. For $g$ in all three configurations to be covered, three additional $C_{k+1}$ segments must exist. Those segments exist in $C_{k+1}$ if the $C_{k}$ segments in path $t$ exist. Path $t$ exists only if the segments denoted as $u_{1}$ and $v_{1}$ exist $\left(u_{1}\right.$ starts at the vertex $U$, and $v_{1}$ ends at the vertex $V$ ).

Since the $L$ and $F$ vertices in $C_{k}$ turn respectively into $B$ and $L$ in $C_{k+1}$, the originally covered OSP $g$ in an $L B$ is a $B L$, in a $B L$ it is an $L B$, and in an $L L$ one $L B$ (towards $U$ ) and one $B L$ (towards $V$ ). By Theorem 3.6 the covered area initiated by $g$ grows in $C_{k+2}$ and $C_{k+3}$ into the same basic shapes, only contracted by $1 / \sqrt{2}$. 

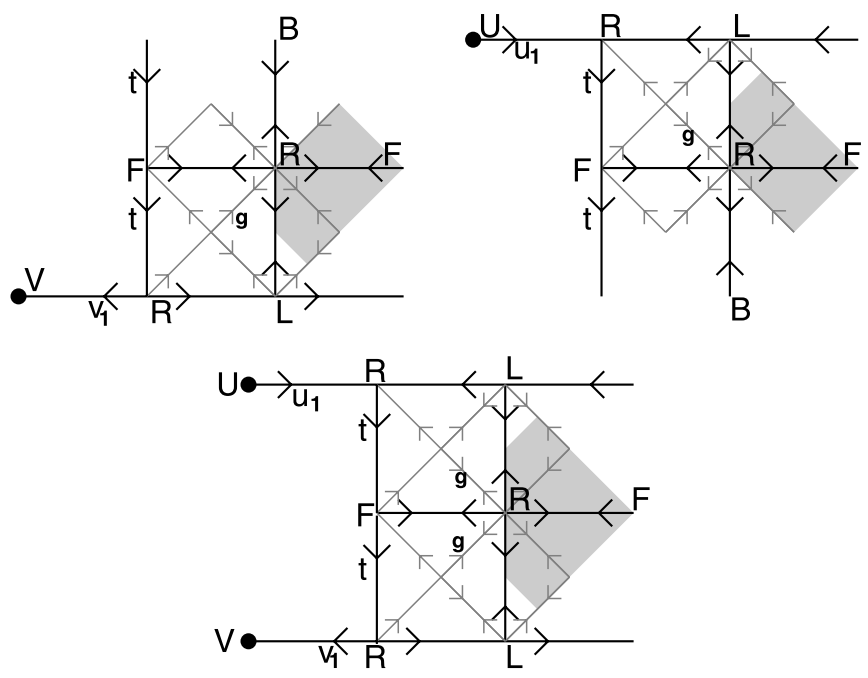

Fig. 11 The originally covered OSP $g$ needed for growth of a $L B, B L$ and $L L$

Fig. 12 First step of tail building
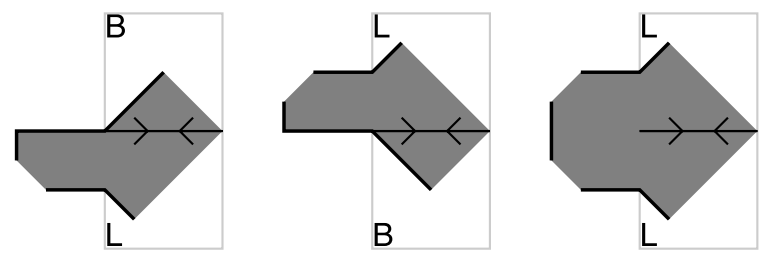

Fig. 13 A tail

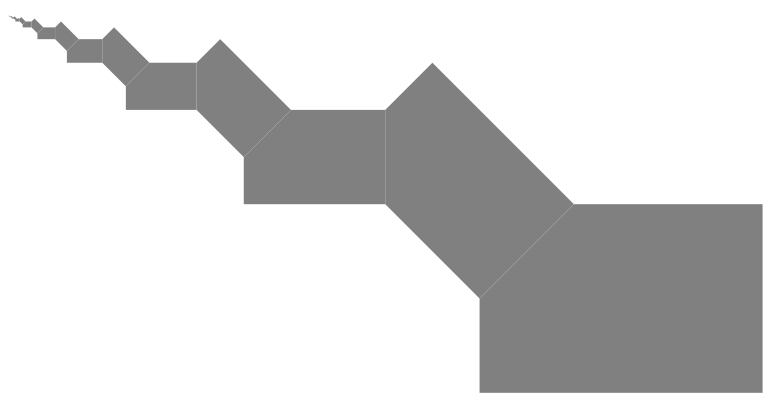

Figure 12 shows the covered shapes obtained in $F^{k+3}(T)$ if $u_{1}$ and $v_{1}$ exist. In $L B$ and $B L$ the $C_{k+1}$ OSP perpendicular to $g$ at its $F$ end cannot be covered since a $C_{k}$ segment with end at the $B$ vertex is in the forbidden path, and thus even if segments $t$ exist, two $C_{k+1}$ segments that must exist if that OSP is covered are absent. Hence, except for one $C_{k+3}$ segment, all segments on the edges of the shape are boundary segments (solid black lines in Fig. 12).

This process can continue creating a tail of contracted basic shapes alternating between a $B L$ shape and an $L B$ shape as demonstrated in Fig. 13. 
Fig. 14 Tail tip segments of $U$ and $V$ tail tips
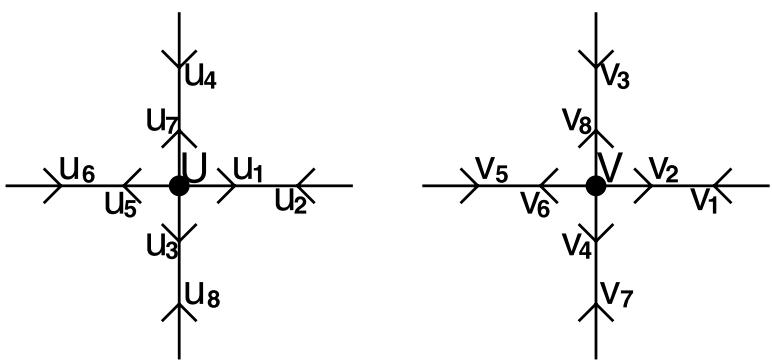

We refer to the number of basic shapes in a tail as its tail length and denote the structures obtained as $B n L, L n B$, and $L n, m L$, where $n$ and $m$ are the tail lengths. Every pair of consecutive basic shapes in a tail is obtained from the previous pair by a contraction by $1 / 2$. The points $U$ and $V$ are the fixed points of these contractions and the tip of the tail in case it is infinite.

For each step of this tail building to go on, the segments equivalent to $u_{1}$ and $v_{1}$ must exist. Note in Fig. 11 that for an $L B(B L)$, the first tail shape is a $B L(L B)$, and its $U(V)$ point is at the $V(U)$ point of the $L B(B L)$. For its $u_{1}\left(v_{1}\right)$ segment to exist so that there will be a second basic shape in the tail, there must be a $C_{k}$ segment opposite to $v_{1}\left(u_{1}\right)$. That segment is denoted $v_{2}\left(u_{2}\right)$, so the existence of $v_{1}$ and $v_{2}$ $\left(u_{1}\right.$ and $\left.u_{2}\right)$ ensures a tail with length 2 for an $L B(B L)$.

Every odd (even) basic shape in a tail is parallel to the first (second) one. Thus, for the $p$ basic shape in a tail to exist, there must be a $C_{k+p-1}$ segment parallel to $v_{1}$ or $u_{1}$ if $p$ is odd and a $C_{k+p-2}$ segment parallel to $v_{2}$ or $u_{2}$ if $p$ is even. This requires the existence of $C_{k}$ segments denoted by $v_{p}$ or $u_{p}$ obtained by rotation by $p$ right angels counterclockwise from $v_{1}$ and $u_{2}$ and clockwise from $v_{2}$ and $u_{1}$, as demonstrated in Fig. 14.

For $p>8$, these same $C_{k}$ segments ensure the existence of the required segments, so that if the first $p-1$ shapes in a tail exist for $p=8 i+j$ if $u_{j}$ or $v_{j}$ exist, then the $p$ shape in the tail exists. Thus, for any $p<9$, if $u_{p}$ (or $v_{p}$ ) is absent while all the segments $u_{1}$ up to $u_{p-1}$ (or $v_{1}$ up to $v_{p-1}$ ) exist, then the tail length is finite and given by $p-1$, while if all eight segments exist, the tail length is infinite. There are thus only nine possible tail lengths for any tail, and this leads to our main result.

Theorem 3.8 The number of shapes of the interior components of the Levy dragon is finite.

Proof By Theorem 3.7 there is a finite number of initial sets of edge connected covered triangles (8 possible configurations). For each of those, there is a finite number of tails (up to 6). Whatever the tail tip type is, there is a finite number of possible tail lengths (9), hence the number of possible shapes is finite.

To determine the possible interior components shapes, we first show that the possible tail lengths for the tails of an $L B, B L$, or $L L$ are much more limited than those nine possible lengths. Next, the tail tip types for all tails in all the configurations obtained for different $Z$ values in Theorem 3.7 are determined. Finally, some shapes 
Table 1 Tail tip consecutive segments pairs and possible tail lengths

\begin{tabular}{lll}
\hline Tail tip type & Segment pairs & Possible tail lengths \\
\hline$R$ & $\left(v_{1}, v_{8}\right),\left(v_{2}, v_{7}\right),\left(v_{3}, v_{6}\right),\left(v_{4}, v_{5}\right)$ & $0,1,2,3, \infty$ \\
$F$ & $\left(v_{7}, v_{8}\right),\left(v_{6}, v_{1}\right),\left(v_{3}, v_{4}\right),\left(v_{5}, v_{2}\right)$ & $0,1,2,6, \infty$ \\
$L$ & $\left(v_{5}, v_{8}\right),\left(v_{6}, v_{7}\right),\left(v_{1}, v_{4}\right),\left(v_{2}, v_{3}\right)$ & $0,1,4,5, \infty$ \\
$B$ & $\left(v_{3}, v_{8}\right),\left(v_{4}, v_{7}\right),\left(v_{5}, v_{6}\right),\left(v_{1}, v_{2}\right)$ & $0,2,3,4, \infty$ \\
\hline
\end{tabular}

Table 2 Possible tail lengths for a $L B$ or $B L$

\begin{tabular}{lll}
\hline Tail tip type & Possible tail length & + path exists \\
\cline { 2 - 3 } & + path absent & $3, \infty$ \\
$R$ & 2 & $1,2, \infty$ \\
$F$ & 0 & $0,1, \infty$ \\
$L$ & Impossible & $0, \infty$ \\
\hline
\end{tabular}

that could exist by these conditions are proved to be impossible because of some dependencies between paths that provide tail tip segments to different tails within the same configuration.

Lemma 3.9 The tail lengths of an $L B$ and a BL are limited to the values in Table 2 depending on the tail tip type and existence or absence of the + path.

Proof The eight tail tip segments are always in four pairs of consecutive segments depending on the tail tip type. The two segments of such a pair either both exist, or both are absent. Hence, there are only three possible finite nonzero tail lengths for any tail tip type shown in Table 1.

Actually the possibilities for the tail tips in a given $L B$ or $B L$ configuration are more limited since some of the tail tip segments are always provided by paths within the minimal configuration continued towards the tail tips. Figure 15(a) demonstrates how by using the $R$ and $F$ vertices within the $L B$ configuration and $C_{k}$ and $C_{k-1}$ squares we can determine the $R, F$, and $L$ vertices on paths towards the $V$ tail tips. Thus, for the $V$ tail tip of any LB, $v_{7}$ and $v_{8}$ always exist. In addition, $v_{6}$ is in the + path which for some configurations is forbidden and for some optional. As demonstrated in Fig. 15(b), this holds also for any BL and its $U$ tail tip segments. Hence, the possible tail lengths for an $L B$ or $B L$ are those in Table 2.

Lemma 3.10 The only possible tail length combinations for the double tail of an $L L$ are $L 0,0 L, L \infty, 1 L, L 1, \infty L$, and $L \infty, \infty L$.

Proof As demonstrated in Fig. 15(c), continuations of paths of the minimal $L L$ configuration provide $v_{7}$ and $v_{8}$ for the $V$ tail tip and $u_{7}$ and $u_{8}$ for the $U$ tail tip. $U, V$, and the two $L$ vertices are on the four corners of a $C_{k-2}$ square; hence either $U=L$ 


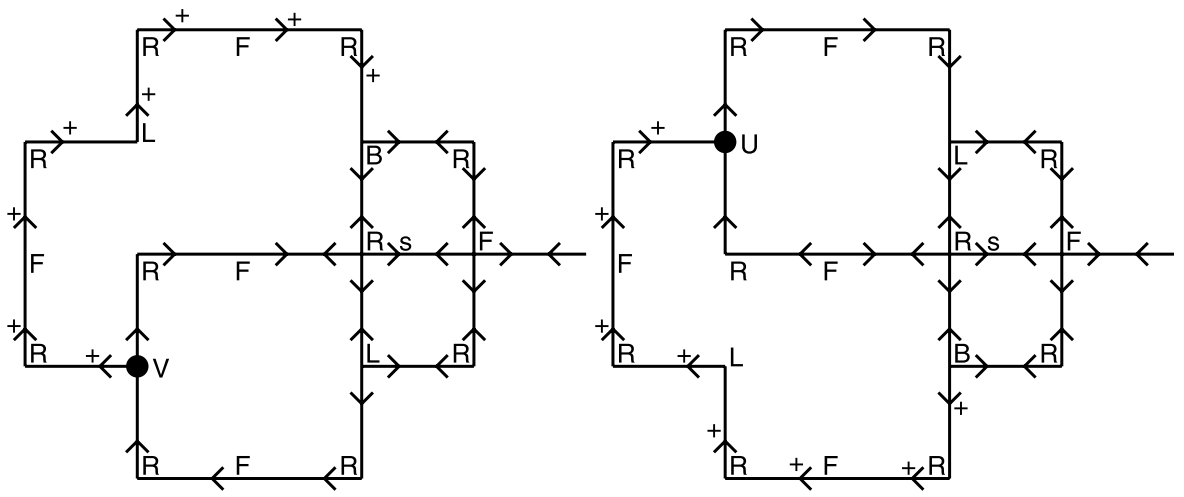

(a)

(b)

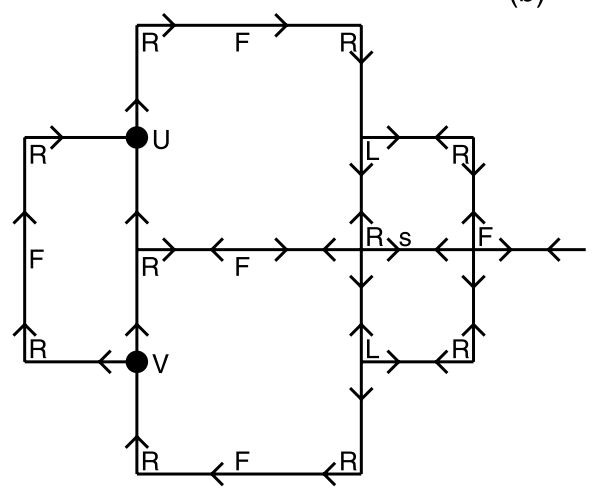

(c)

Fig. 15 Tail tip segments provided by paths in the originally covered configuration and the + path

and $V=B$, or $U=B$ and $V=L$. In the first case, $u_{6}$ and $u_{7}$ are on the same path, while in the second case, $v_{7}$ and $v_{6}$ are on the same path. But $v_{6}$ and $u_{6}$ are on the same path shown in Fig. 15(c); hence $u_{6}$ and $v_{6}$ always exist for any LL. $u_{1}$ and $v_{1}$ are on the same path; hence either both are absent and the double tail is $L 0,0 L$, or both exist, in which case both tails have nonzero length. If $U=L, V=B$, and $u_{2}$ is absent, the double tail is $L \infty, 1 L$, while if $U=B, V=L$, and $v_{2}$ is absent, the double tail is $L 1, \infty L$. In case $U=L$ if $u_{2}$ also exists or in case $V=L$ if $v_{2}$ also exists, then the double tail is $L \infty, \infty L$.

There are six points that are tail tips in all or some of the eight cases in Fig. 10. These six tail tips, denoted by $V_{1}, U_{1}, U_{2}, V_{2}, V_{3}$, and $U_{3}$, are emphasized in Fig. 16(a), which is the $C_{k-2}$ configuration from which the $C_{k}$ configuration in Fig. 9 is obtained by replacing every segment in Fig. 16(a) by a $C_{2}$. The segments in Fig. 16(a) are denoted by the labels of the segments that replace them in Fig. 9 and are extended up to the tail tips. The type in $C_{k-2}$ of a vertex with type $X$ in $C_{k}$ is denoted by $X^{*}$.

Since $Z$ is at a corner opposite a $B$ in a $C_{k-2}$ square, we have that if $Z \neq B$ it was created before $C_{k-3}$, while if $Z=B$, it might be that $Z=B^{[k-3]}$ and the other $B$ 


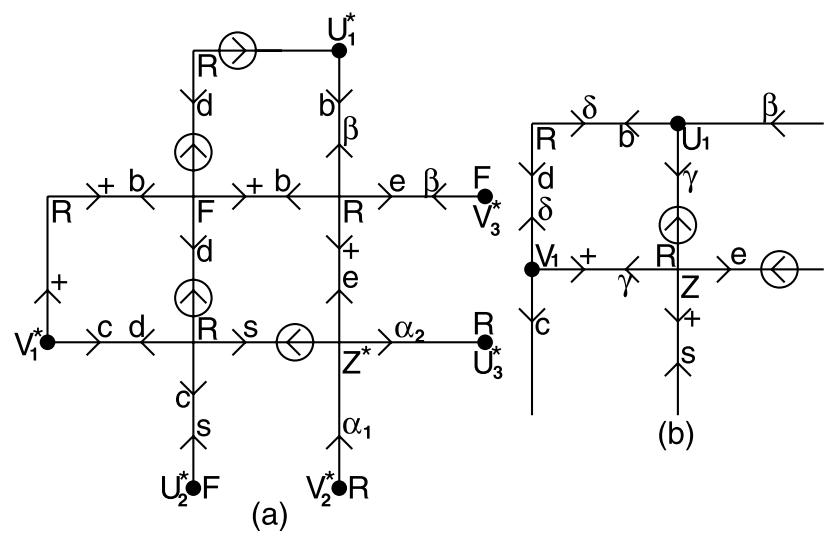

Fig. 16 (a) The six possible tail tips in the $C_{k-2}$ configuration. (b) The $C_{k-4}$ configuration in case $Z=R^{[k-4]}$

created before $C_{k-3}$. But these two possibilities lead to configurations that are related by a rotation and thus will lead to the same shapes; hence for the purpose of finding all shapes, we can assume that $Z$ was created before $C_{k-3}$. Hence, $Z=R^{[k-4 q]}$, or $Z=B^{[k-3-4 q]}$, or $Z=L^{[k-2-4 q]}$ for some integer $q>0$.

Proposition 3.11 The tail tip type for all the tails of the configurations in Theorem 3.7 are determined.

Proof First, consider the case $Z=R^{[k-4]}$. Figure 16(b) shows the $C_{k-4}$ configuration in this case. Either $V_{1}$ or $U_{1}$ is an $F^{[k-5]}$. By Theorem 3.7 the possible shapes are $L B$ and $L B L$. For the $L B$ shape, the $\beta$ path is absent, but if $U_{1}=F$, then $\beta$ is in the $b$ path; hence $U_{1} \neq F$ and $V_{1}=F$. For an LBL shape, $\beta$ exists, and the + path is absent. $U_{1} \neq L$, so that $b$ is not in the forbidden path, and $V_{1} \neq L$, so that $d$ is not in the + path. Since at least one out of $V_{1}$ and $U_{1}$ is $F$, the possibilities for $\left(V_{1}, U_{1}\right)$ are $(F, F),(F, B),(F, R),(B, F),(R, F)$. The last two lead to configurations that are reflected versions of the previous two; hence for the purpose of determining the possible shapes, we can assume that $V_{1}=F$ and $U_{1}$ is $F, B$, or $R$.

For the rest of the possibilities, since $\mathrm{Z}$ was created before $C_{k-4}$, then $V_{1}=U_{1}=$ $R^{[k-4]} . U_{2}, V_{2}$, and $Z$ are on three corners of a $C_{k-2}$ square with an $L^{[k-2]}$ in the last corner; hence $V_{2}=L^{[k-2]}$ and $U_{2}=B^{[k-3]}$. Also $V_{3}, U_{3}$, and $Z$ are on three corners of another $C_{k-2}$ square with an $L^{[k-2]}$ in the last corner; hence $U_{3}=L^{[k-2]}$ and $V_{3}=B^{[k-3]}$. Thus, all six tail tip types are known.

Figure 17 shows the $C_{k-2}$ configuration with the paths extended up to the tail tips and the additional paths $\gamma, \delta, \mu, v, \eta$, and $\chi$ that might provide the rest of the tail tip segments.

Lemma 3.12 If $Z=R^{[k-4]}$, then the shape L2B is impossible. 
Fig. 17 The $C_{k-2}$ configuration in case $Z$ was created before $C_{k-4}$

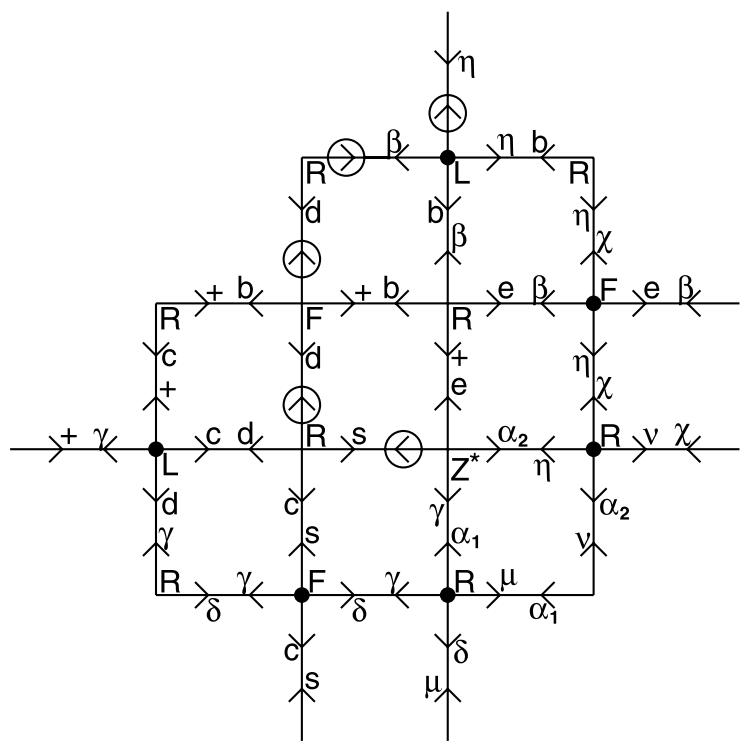

Proof By Proposition 3.11 in this case $V_{1}=F$. Two paths, $\gamma$, which provides $v_{2}$ and $v_{5}$, and $\delta$, which provides $v_{3}$ and $v_{4}$, are added in Fig. 16(b) and extended up to $U_{1}$. If $U_{1}=L$, then $b$ is in the forbidden path, and hence $U_{1} \neq L$. If $\gamma$ exists, then also $U_{1} \neq B$; otherwise $\gamma$ is in the forbidden path. Thus, if $\gamma$ exists, then $U_{1}=R$, but then $\delta$ is in the same path as $\gamma$, so both of them exist. For an $L 2 B$ shape, $\gamma$ exists and $\delta$ is absent; hence it is impossible in this case.

Note that $L 2 B$ is possible in other configurations $Z=R^{[k-4 q]}$ with $q>1$ and $Z=L^{[k-2-4 q]}$.

Lemma 3.13 If $Z=B$, the shape $B 0 L \infty B$ is impossible.

Proof For $B 0 L \infty B$ to exist, in case $Z=B$, the + path must exist, but the $\delta$ path must be absent. Consider the vicinity of $Z$ when it was created in $C_{k-3-4 q}$ obtained from its vicinity in $C_{k}$ and demonstrated in Fig. 18(a) for $q$ even and Fig. 18(b) for $q$ odd. In both cases the square with corners at $P, Q, S$, and $T$ is a $C_{k-4-4 q}$ square and hence in $C_{k-3-4 q}$ either $P=Q=F$ and one out of $S$ and $T$ is $L$, or $S=T=F$ and one out of $P$ and $Q$ is $L$. If $Q=F$, then in both cases $\delta$ is in the $c$ path, so it always exists. If $S=T=F$, then in the even $q$ case, if $P=L$, the $\beta$ path is in the $e$ path, but $\beta$ is absent, hence $P \neq L$ and $Q=L$, and thus $\delta$ is in the $\gamma$ path which is in the + path, while in the odd $q$ case, if $P=L$, then $\beta$ is in the + and $\gamma$ path, and if the + path exists, $P \neq L$ and $Q=L$, and $\delta$ is in the $e$ path. Thus, in both cases, if the + path exists, then $\delta$ exists, so $B 0 L \infty B$ is impossible.

Lemma 3.14 If $Z=L$ the shape $L 2 B 2 L \infty, 1 L 1, \infty$ l is impossible. 
Fig. 18 The $C_{k-3-4 q}$ configurations in case $Z=B$ (a) $q$ even (b) $q$ odd

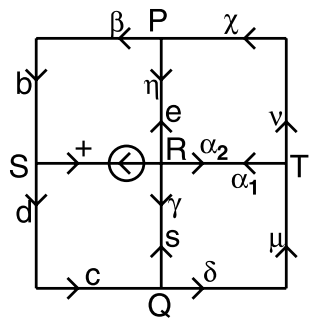

(a)

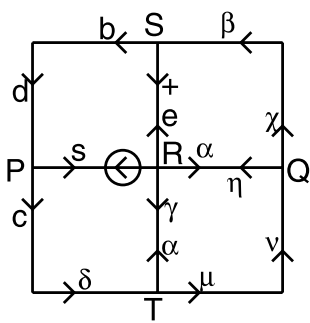

(a)

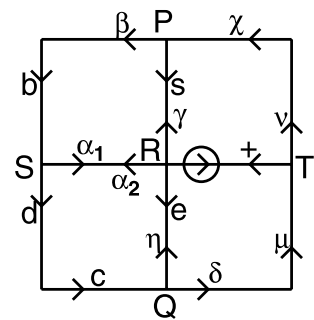

(b)

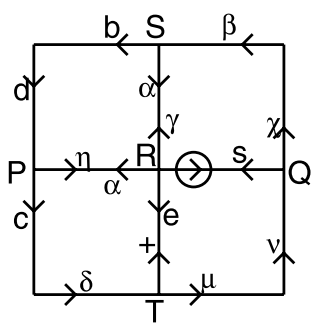

(b)
Fig. 19 The $C_{k-2-4 q}$ configurations in case $Z=L$ (a) $q$ even (b) $q$ odd

Proof Consider the vicinity of $Z$ when it was created in $C_{k-2-4 q}$, as demonstrated in Fig. 19(a) for $q$ even and Fig. 19(b) for $q$ odd. In both cases the square with corners at $P, Q, S$, and $T$ is a $C_{k-3-4 q}$ square, and hence in $C_{k-2-4 q}$ either $P=Q=F$ or $S=T=F$. In both cases, if $T=F$, then $\delta$ and $\mu$ are in the same path, and if $Q=F$, then $v$ and $\chi$ are in the same path, thus it never happens that $\chi$ and $\delta$ exist while $v$ and $\mu$ are absent, and thus $L 2 B 2 L \infty, 1 L 1, \infty L$ is impossible.

Theorem 3.15 Each interior component of the Levy dragon is similar to one out of the following 23 shapes:

$5 L B$ shapes: $L 0 B, L 1 B, L 2 B, L 3 B$, and $L \infty B$;

$4 L B L$ shapes: $L O B 0 L, L 2 B 0 L, L 4 B 0 L$, and $L 2 B 2 L$;

$3 L L B$ shapes: $L \infty, \infty L 2 B, L 1, \infty L 2 B$, and $L 0, O L 2 B$;

$3 B L B$ shapes: $B \infty L \infty B, B 2 L 4 B$, and $B 0 L 2 B$;

$3 L B L B L$ shapes: $L 2 B 2 L 0 B 4 L, L 2 B 2 L 4 B 4 L$, and $L 2 B 2 L 0 B 0 L$;

5 LBLLL shapes: $L 2 B 2 L 0,0 L 0,0 L, L 2 B 2 L \infty, 1 L 0,0 L, L 2 B 2 L \infty, \infty L 0$, $0 L, L 2 B 2 L \infty, 1 L \infty, \infty L$, and $L 2 B 2 L \infty, \infty L \infty, \infty L$.

Proof Using all the initial configurations in Theorem 3.7 with the tail tip types determined in Proposition 3.11 and the corresponding tail lengths in Lemma 3.9 and Lemma 3.10 and removing the impossible shapes in Lemmas 3.12, 3.13, and 3.14, these 23 shapes are the only possible interior components shapes of the Levy dragon.

These 23 possible shapes are demonstrated in Fig. 20. 


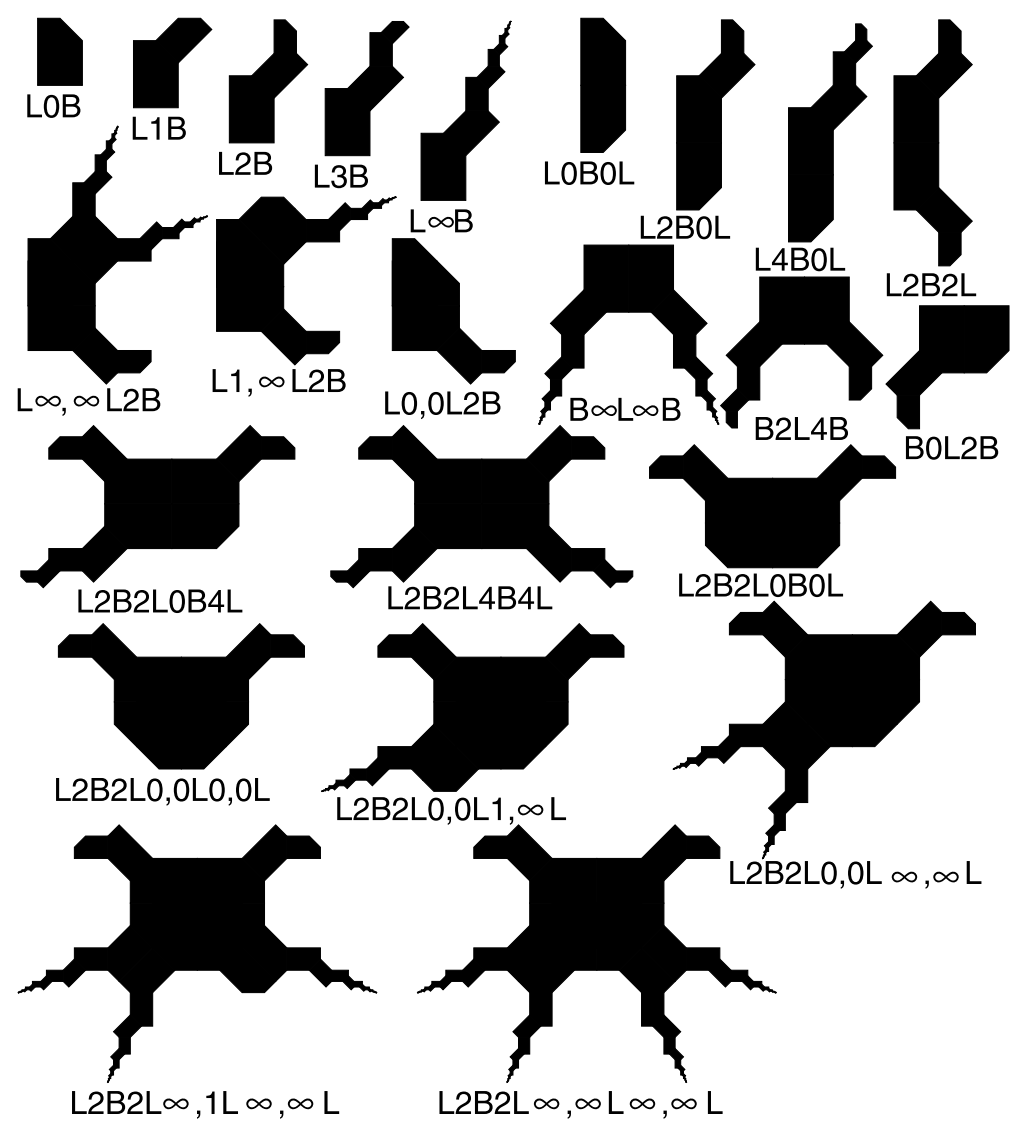

Fig. 20 The 23 possible shapes of interior components of the Levy dragon

By using a program that draws the $F^{k}(T)$ triangles differentiating between covered and not covered triangles one can see that all these 23 possible shapes appear. One representative of each shape is initiated up to $C_{35}$ within distances less than $3 l_{14}$ from an $L O B O L$ initiated in $C_{14}$.

The method of this paper can be used to determine many other geometric properties of the Levy dragon. For example, replacing existing segments by absent segments and vice versa, one can determine that the possible shapes of the holes of the Levy dragon are the same 23 shapes. By using a similar program one can see that there are holes with all those shapes. Finding those hole shapes is a much easier task than finding the interior components shapes since their configurations appear much earlier in the $C_{k}$ sequence. Some of the holes are thus big enough to be seen in Fig. 1. The first one to appear in $C_{7}$ is the configuration of an $L 2 B 2 L \infty, \infty L \infty, \infty L$ hole seen in Fig. 1 at the center of the Levy dragon. 


\section{References}

1. Bailey, S., Kim, T., Strichartz, R.S.: Inside the Levy dragon. Am. Math. Mon. 109, 689-703 (2002)

2. Duvall, P., Keesling, J.: The Hausdorff dimension of the boundary of the Levy dragon. Int. J. Math. Math. Sci. 20, 627-632 (1997)

3. Deng, D.W., Ngai, S.M.: Vertices of self-similar tiles. Ill. J. Math. 49, 857-872 (2005)

4. Edgar, G.A.: Classics on Fractals, pp. 181-239. Addison-Wesley, Reading (1993);

5. Hutchinson, J.E.: Fractals and self similarity. Indiana Univ. Math. J. 30, 713-747 (1981)

6. Lévy, P.: Les courbes planes ou gauches et les surfaces composé de parties semblales au tout. J. École Polytech. 227-247, 249-291 (1938)

7. Ngai, S.M., Nguyen, N.: The Heighway dragon revisited. Discrete Comput. Geom. 29, 603-623 (2003)

8. Ngai, S.M., Tang, T.M.: Topology of connected self-similar tiles in the plane with disconnected interior. Topol. Appl. 150, 139-155 (2005)

9. Strichartz, R.S., Wang, Y.: Geometry of self-affine tiles I. Indiana Univ. Math. J. 48, 1-24 (1999) 